\title{
Qué gana STEM con la gamificación
}

\author{
Mercedes Fuentes Hurtadoa - Juan González Martínez
}

Resumen: En el ámbito STEM (science, technology, engineering, maths), cuyo objetivo es formar de modo integrado a ciudadanos capaces de enfrentarse a los retos del siglo XXI, la gamificación educativa es un recurso cada vez más frecuente en todos los niveles educativos y supone un elemento clave para mejorar el proceso de enseñanza-aprendizaje. Por medio de un análisis de la literatura, se justifica cómo la gamificación aporta beneficios al estudio de las materias de STEM en educación secundaria y se confirma su potencialidad en el aprendizaje significativo, el aumento de la motivación y en el desarrollo de las competencias transversales.

Palabras clave: aprendizaje; competencias transversales; gamificación; motivación.

Recibido: 13 octubre de 2018 - Evaluado: 05 mayo de 2019 - Aceptado: 10 mayo de 2019

a Universitat Rovira i Virgili, Facultad de Ciencias de la Educación y Psicología. Tarragona. E-mail: mfuenteshurtado@gmail.com

b Serra Húnter Fellow, Universitat de Girona, Facultat d'Educació i Psicologia, Departament de Pedagogia. Plaça de Sant Domènech, 9, 17004, Girona, Despatx 240. E-mail: juan.gonzalez@udg.edu 


\section{What Does STEM Gain with Gamification?}

Abstract: In the field of science, technology, engineering, and mathematics (STEM), whose objective is to train citizens capable of facing the challenges of the $21^{\text {st }}$ century, educational gamification is an increasingly frequent resource at all educational levels and is a key element to improve the teachinglearning process. Through a literature review, it is justified how gamification brings benefits to the study of STEM in high school education and confirms its potential in meaningful learning, increased motivation, and the development of cross-sectional skills.

Keywords: Learning; cross-sectional skills; gamification; motivation.

\section{O que o STEM ganha com a gamificação?}

Resumo: O STEM (science, technology, engineering, maths) tem como objetivo formar, de modo integrado, cidadãos capazes de enfrentar os desafios do século XxI; nesse sentido, a gamificação da educação é um recurso cada vez mais frequente em todos os níveis educativos e supõe um elemento-chave para melhorar o processo de ensino e aprendizagem. Por meio de uma análise da literatura, justifica-se como a gamificação é benéfica para o estudo das matérias de STEM na educação secundária e confirma-se sua potencialidade na aprendizagem significativa, no aumento da motivação e no desenvolvimento das competências transversais.

Palavras-chave: aprendizagem; competências transversais; gamificação; motivação. 


\section{Introducción}

Tradicionalmente, en los centros de educación secundaria las asignaturas se imparten de manera compartimentada y se organizan —incluso las del mismo ámbito- de forma aislada de acuerdo con los currículos establecidos (Von Garnier, 2010). En este sentido, y a pesar de la distancia temporal, es aún vigente la visión de Ferguson (1980, p. 324): “la escuela fracciona el conocimiento y la experiencia en asignaturas, reduciendo incesantemente los todos a las partes, las flores a los pétalos, la historia a sucesos, sin preocuparse nunca por restablecer la continuidad", lo cual es una de las consecuencias de ese abordaje atomizado de las materias, sin relación entre sí en su despliegue curricular.

Esto ocurre especialmente en las materias del ámbito científico y tecnológico (en adelante сут), las cuales, en la etapa de la secundaria, se corresponden, principalmente, con matemáticas, física y química, biología, geología y tecnología, cuyos contenidos están relacionados de forma inherente, pero se organizan de manera independiente. Así, los estudiantes llegan a considerar que los contenidos de estas asignaturas no están conectados entre sí y mucho menos con su entorno más cercano; es decir, que los aprendizajes que adquiere el alumnado son también inconexos, desagregados y, en muchos casos, descontextualizados de la realidad más inmediata. Además, según Vázquez y Manassero (2009), esto acarrea que un alto porcentaje de estudiantes desarrolle actitudes negativas, tales como el aburrimiento, el desinterés o incluso el rechazo en relación con estas asignaturas.

En efecto, Marbà y Márquez (2010) evidencian que el interés del alumnado por las asignaturas de сут disminuye de manera progresiva a lo largo de los años de escolarización, desde primaria hasta el final de la secundaria. En opinión de los estudiantes, esto se da en razón a su dificultad y la poca relación con su entorno, lo que viene a verificar la afirmación de Von Garnier (2010), según la cual el alumnado encuentra poco sentido en los contenidos de esta etapa educativa. Lo anterior, de manera inevitable, repercute negativamente en su propia actitud ante el aprendizaje, en la calidad de esos aprendizajes y, en definitiva, a mediano y largo plazo, en el futuro de nuestra sociedad.

Además de las consecuencias más directas (como, por ejemplo, el poco rendimiento del aprendizaje o la escasa conexión de las competencias adquiridas entre sí), esto también se traduce en un bajo impacto en términos de vocaciones científicas, pues los alumnos sienten un desapego hacia los estudios del ámbito сут que les retrae de considerar las carreras técnicas como una opción para sus estudios superiores; esto es, la denominada "crisis de las vocaciones científicas" (Convert y Gugenheim, 2005).

En este contexto, el movimiento sтем (Duque, Celis y Camacho, 2011) promueve la enseñanza integrada de contenidos de сут y requiere implementar metodologías que permitan motivar al alumnado por medio de un aprendizaje significativo que le permita desarrollar actitudes positivas hacia la сут, de modo que esto se refleje en una mejora de los aprendizajes, de los resultados académicos y en un aumento de las vocaciones científico-tecnológicas.

En busca de esa motivación del alumnado muchos docentes apuestan por recuperar el juego y aprovechar sus beneficios en el ámbito educativo, de modo similar a como se está realizando con éxito en otros entornos no lúdicos tales como el económico o el empresarial (Cortizo Pérez et al., 2011; Ovallos, Villalobos, De la Hoz y Maldonado, 2016). En este sentido, Herranz y Colomo-Palacios (2012), así como Werbach (2012), recuerdan que los juegos provocan casi cualquier tipo de emoción imaginable, reforzando la curiosidad, la competitividad o la felicidad. Además, por tanto, se puede pensar que una metodología gamificada, es decir, aquella que emplea elementos y el pensamiento de juego en contextos de no juego (Foncubierta y Rodríguez, 2006), contextualizada en el ámbito STEM, proporcionará las herramientas necesarias para ofrecer al alumnado una enseñanza global, significativa, que conecte con sus emociones $y$, por ende, adecuada al propósito de conseguir los objetivos educativos del profesorado. 


\section{Marco teórico}

El desapego por el conocimiento que sienten los estudiantes de secundaria se debe, según Von Garnier (2010, p. 109), al error que se comete "educando a los jóvenes como se hacía en la era industrial y no en la época de la información y los conocimientos”. Los jóvenes de hoy, protagonistas de la revolución digital (González y López, 2011), requieren que los procesos educativos se aborden desde una perspectiva renovada (Melchor, 2012). Así, la transdisciplinariedad y el enfoque por proyectos sirven al alumnado de hoy para entender el mundo en el que viven y aplicar sus conocimientos y habilidades en distintos contextos (Von Garnier, 2010; Trujillo, 2012).

\section{La educación integrada stem}

No ha sido hasta el 2011 cuando se ha acuñado el acrónimo sтем (Duque et al., 2011) para hacer referencia a las asignaturas de ciencias, tecnología, matemáticas e ingeniería, considerándolas fundamentales en las sociedades tecnológicamente avanzadas y reconocidas en las políticas educativas de los países desarrollados, donde existe una preocupación por la disminución de estudiantes que cursan estudios universitarios de ciencias e ingenierías, y donde también ha crecido el interés por la formación no solo de futuros ingenieros, sino también de ciudadanos que deben alfabetizarse tecnológicamente para afrontar los retos del siglo XXI (Duque et al., 2011).

La iniciativa STEM pretende aprovechar las similitudes de las asignaturas de сут para desarrollar enfoques transdisciplinares que incorporen contextos y situaciones de la vida cotidiana y utilicen, además, las herramientas tecnológicas necesarias, en línea con los objetivos educativos marcados por la Unión Europea para el 2020. Así, tal y como afirman Stohlmann, Moore y Roehrig (2012), la educación integrada STEM es una manera de hacer el aprendizaje más conectado, relevante y significativo para los estudiantes, en un esfuerzo por combinar las materias del ámbito con un abordaje único, no disgregado, basado en las conexiones entre dichas materias y los problemas de la vida real. Esto, por ende, propiciará también actitudes positivas de curiosidad e interés que lleven al alumnado a apreciar las ciencias y la tecnología (Vázquez y Manassero, 2009). Así, y en segunda instancia, se espera aumentar el número de graduados en titulaciones сут, además de reducir el desequilibrio entre hombres y mujeres (Consejo de Europa, 2003), mejorar la crisis de las vocaciones científicas desde edades tempranas y la cualificación científico-tecnológica de la ciudadanía (Quigley, Herro y Jamil, 2014).

La evolución del concepto STEM ha derivado en la actualidad en STEAM, sigla en la que la "A" hace referencia a la inclusión del arte y las humanidades en el aprendizaje transdisciplinar (Quigley et al., 2014), del que empiezan a proliferar referencias en la bibliografía asociada.

\section{Del juego a la gamificación educativa}

El concepto gamificación proviene de la palabra inglesa gamification, y aunque como se ha dicho se ha aplicado con éxito en otros campos (Cortizo Pérez et al., 2011), es relativamente nuevo en educación; no fue sino hasta el 2008 cuando comenzó a proliferar en los medios digitales. Según Melchor (2012), a partir del 2010 se adoptó tanto el término como el concepto de forma generalizada, si bien es indudable que antes se había abordado con cierta profusión el juego con finalidades educativas y usos tecnológicos, las cuales están en la base del discurso actual sobre gamificación (Luis-Pascual, 2015).

A partir de la idea de jugar, Carpena, Cataldi y Muñiz (2012, pp. 633-634) recuerdan que "el primer modo eficaz de asir la realidad es a través del juego. Jugando aprendemos a comer, a caminar, a hablar, a expresarnos artísticamente, a generar lazos sociales, a reconocer y asimilar nuestro entorno". Por su parte, Da Silva (2014), citando a Kischimoto (1993), comenta que el juego ha formado parte de la educación y ha demostrado su eficacia en todos los periodos históricos, así como ha evolucionado hasta hoy con el desarrollo de los denominados "serious games" (Deterding, 2012). Zichermann y Cunningham (2011), Kapp (2012), 
y Díaz y Troyano (2013), entre otros, van más allá y proponen una definición de gamificación que consiste en llevar al contexto educativo técnicas y mecánicas de juegos que atraen y estimulan a la persona que participa en la experiencia con el fin de promover el aprendizaje, y así incitar a la acción para resolver problemas. Es ahí cuando hablamos, por ejemplo, de elementos propios de los juegos tales como los puntos, los niveles o las clasificaciones, que proporcionan placer, diversión y motivación a los aprendientes.

Todo esto genera efectos positivos en el aprendizaje del alumnado. Así, Hamari, Koivisto y Sarsa (2014) evidencian que incluir la mecánica de juegos en educación mejora el disfrute y la satisfacción del alumnado por la actividad educativa, así como la voluntad por participar de forma activa, con una mayor implicación y control del tiempo. Debe tenerse en cuenta, tal como lo recuerdan Posada (2013) y Martín Martín (2017), la importancia de llegar a un equilibrio entre lo lúdico y lo formativo, a fin de que los aspectos más positivos de la gamificación en el aula (motivación, alfabetización tecnológica, mentalidad multitarea, trabajo en equipo e instrucción personalizada) repercutan en los aprendizajes. Por su parte, Melchor (2012) afirma que la introducción de los elementos propios de la gamificación no tiene por qué llevarse a cabo solo a través de juegos concretos, sino que existen otras muchas maneras diferentes de gamificar el aula mediante ejercicios, trabajos o proyectos en los que se introduzcan determinados elementos propios de los juegos como lo son los logros, las recompensas, la competición, las clasificaciones o el continuo feedback que, por otra parte, también están presentes en los videojuegos actuales (Sánchez-Aparicio, 2014), y son una de las formas habituales de entretenimiento juvenil (Martín del Pozo, 2013).

Los resultados de todas estas experiencias, frecuentes en el ámbito stem, llevan a Pérez-Manzano y Almela-Baeza (2018) a señalar que la gamificación es posiblemente la herramienta metodológica más relevante para potenciar la motivación del alumnado cuando se busca la mejora de los aprendizajes y de los resultados académicos.

\section{Hipótesis y objetivos}

A partir de la hipótesis según la cual la enseñanza de sтем en secundaria se ve beneficiada con la introducción de la gamificación como herramienta metodológica, en este artículo nos proponemos como objetivo, precisamente, justificar a partir de la revisión de la literatura en la que se documentan experiencias docentes por qué resulta beneficioso integrar la gamificación en el ámbito STEM cuando se busca la mejora del proceso de enseñanza-aprendizaje.

\section{Metodología}

A fin de alcanzar el objetivo marcado, se ha realizado un estudio cualitativo basado en el uso de la técnica del análisis documental, el cual se centra en recopilar, estudiar y analizar diferentes propuestas didácticas que emplean la gamificación en STEM y cuáles han sido los resultados obtenidos. Este análisis documental se basa en el rastreo e inventario de los documentos disponibles, su posterior clasificación - al seleccionar los más pertinentes para la investigación- $y$, finalmente, una lectura profunda, cruzada y comparada que se les aplica a fin de construir una síntesis, de acuerdo con los pasos descritos por Bisquerra (2009). Se explican, a continuación, de forma pormenorizada, las fases del estudio documental realizado.

\section{Rastreo e inventario de los documentos disponibles}

La presente reflexión sobre STEM y gamificación se estructura con base en una selección de textos de la literatura del ámbito obtenidos mediante la búsqueda selectiva de documentos que relacionan ambas temáticas a partir de palabras clave en los repositorios accesibles (WoK, iCercador, Google Schoolar, ERIC, Scopus). Hasta donde conocemos, ambas disciplinas - STEM y gamificación aplicadas al contexto educativo- en razón a que son innovadoras, no cuentan con un acervo abundante de referencias bibliográficas anteriores a los años 2011 y 2008, respectivamente, por lo que todas las publicaciones relativas a estos dos conceptos son novedosas y de gran actualidad. 


\section{Clasificación, selección y síntesis de los documentos consultados}

Una vez recopilados, los documentos consultados se han clasificado según su temática tal y como se presenta en la Tabla 1. Así, se han establecido tres grandes grupos: documentos relativos a las enseñanzas integradas en el ámbito STEM, documentos relacionados con la gamificación educativa y documentos que muestran experiencias docentes gamificadas para STEM en diferentes escenarios educativos.

Tabla 1. Número de documentos consultados por temática

\begin{tabular}{l|c}
\hline Temática & $\begin{array}{l}\text { N. }{ }^{\circ} \text { de documentos } \\
\text { consultados }\end{array}$ \\
\hline $\begin{array}{l}\text { Ámbito STEM y enseñanzas } \\
\text { integradas }\end{array}$ & 9 \\
\hline Gamificación en educación & 28 \\
\hline Gamificación educativa en STEM & 29 \\
\hline
\end{tabular}

Fuente: elaboración propia.

Este último grupo es el que resulta de mayor interés, ya que se centra en los dos ejes de esta investigación: STEM y gamificación. De esta manera son, por tanto, los documentos que han sido estudiados prioritariamente para justificar los beneficios de la introducción de la gamificación en el ámbito STEM. Los detalles de los documentos más relevantes para esta investigación se indican en el Apéndice A.

Ha resultado interesante poner el foco también en niveles educativos distintos a la secundaria, en los cuales se aprecia una mayor difusión académica del empleo relevante de metodologías gamificadas en STEM que, a veces, es escasa en secundaria. Como apuntábamos, el acervo documental en los repositorios académicos restringido al análisis de experiencias en el nivel de la secundaria es extraordinariamente reducido, en parte por la novedad de la generalización de esta metodología en el ámbito STEM, en parte por una cuestión de falta de tradición de comunicación de las experiencias docentes en secundaria por los canales habituales de la comunicación científica. Ante la eventualidad de armar esta reflexión solo con esos pocos documentos (14), se decidió incluir secundariamente las referencias que se consignan del ámbito universitario, a causa de los indudables puntos en común con el tópico abordado aquí y a pesar de la obvia diferencia de nivel educativo.

\section{Resultados}

\section{Gamificación en stem}

Introducir la gamificación en STEM tiene, como decíamos, el objetivo de potenciar en el alumnado la motivación por adquirir los conocimientos de este ámbito. Así, incorporar elementos del juego a la dinámica de la clase propicia que el alumnado se involucre en su propio aprendizaje y traslade al ámbito educativo el interés que siente al jugar (Werbach y Hunter, 2012).

Estos elementos, característicos de los juegos, se pueden dividir en tres categorías que - del nivel más abstracto al más concreto- son: dinámicas, mecánicas y componentes (Cheong, Filippou y Cheong, 2014). Por una parte, las dinámicas (tales como narrativas, progresiones, emociones, etc.) han de ser consideradas pero no implementadas directamente en los juegos; por otra, las otras dos categorías sí han de serlo, y, por tanto, se presentan en la Tabla 2 ejemplos de elementos del juego pertenecientes a estas dos categorías, de acuerdo con los propuestos por Cheong et al. (2014), que a su vez se basan en los establecidos por Werbach y Hunter (2012).

Tabla 2. Ejemplos de elementos del juego según categorías

\begin{tabular}{l|l}
\hline Categoría & Ejemplos de elementos del juego \\
\hline Mecánicas & $\begin{array}{l}\text { Retos, desafíos, competición, cooperación, } \\
\text { feedback, recompensas, premios. }\end{array}$ \\
\hline Componentes & $\begin{array}{l}\text { Avatares, logros, niveles, puntos, equipos, } \\
\text { insignias, medallas. }\end{array}$ \\
\hline
\end{tabular}

Fuente: elaboración propia.

En específico, en el ámbito STEM, Ortiz-Colón, Jordán y Agredal (2018) revisan un amplio abanico de experiencias de gamificación educativa entre el 2011 y el 2016, y muestran que la introducción en educación de elementos del juego, como, por ejemplo, los indicados en la Tabla 2, influye en la mejora 
de los aprendizajes, en los resultados de las pruebas o en los exámenes, y favorece la motivación y el rendimiento del alumnado. Además, fomenta el buen ambiente en clase y ayuda al alumnado a entender el mundo que lo rodea, al mismo tiempo que desarrolla habilidades tales como la concentración, la concienciación social (Uribe, Utrilla y Santamaría, 2017), la autoestima, la autoconfianza, las habilidades sociales y las competencias personales (Calderón, Petri, Ruiz y Gresse, 2018).

A manera de resumen de los documentos consultados, y con una estructura inspirada en la propuesta por Area y González (2015), adaptada de Nah, Zeng, Telaprolu, Ayyappa y Eschenbrenner (2014), la Tabla 3 presenta experiencias educativas llevadas a cabo en secundaria, en las cuales la gamificación

Tabla 3. Resumen de las experiencias docentes gamificadas de secundaria analizadas

\begin{tabular}{|c|c|c|c|}
\hline $\begin{array}{l}\text { Experiencias } \\
\text { documentadas }\end{array}$ & Beneficios en la enseñanza-aprendizaje & Elementos del juego & $\begin{array}{l}\text { Tecnología con que se } \\
\text { implementa }\end{array}$ \\
\hline Murua (2013) & $\begin{array}{l}\text { - Mejora de la gestión del aula. } \\
\text { - Aumento de la motivación. }\end{array}$ & $\begin{array}{l}\text { - Ranquin de puntos. } \\
\text { - Medallas. } \\
\text { - Insignias. }\end{array}$ & Red social Class Dojo \\
\hline Manzanares (2014) & $\begin{array}{l}\text { - Mejora de la gestión del aula. } \\
\text { - Aumento de la motivación. } \\
\text { - Atención a los diferentes ritmos de aprendizaje. }\end{array}$ & •Insignias. & Red social ClassBadgets \\
\hline $\begin{array}{l}\text { García Velategui } \\
\text { (2015) }\end{array}$ & • Mejora de la gestión del aula. & $\begin{array}{l}\text { - Insignias. } \\
\text { - Ranquin de puntos. } \\
\text { - Medallas. }\end{array}$ & $\begin{array}{l}\text { Redes sociales } \\
\text { ClassBadgets y Class Dojo }\end{array}$ \\
\hline Vega y Cañas (2014) & $\begin{array}{l}\text { - Aumento de la motivación. } \\
\text { - Mejora en la resolución de problemas reales. }\end{array}$ & $\begin{array}{l}\text { - Competición. } \\
\text { - Retos y desafíos. }\end{array}$ & $\begin{array}{l}\text { Robótica con } \\
\text { Lego Mindstorm }\end{array}$ \\
\hline $\begin{array}{l}\text { Ocaña, Romero, Gil y } \\
\text { Codina (2015) }\end{array}$ & $\begin{array}{l}\text { - Aumento de la motivación. } \\
\text { - Mejora en los resultados académicos. }\end{array}$ & $\begin{array}{l}\text { - Competición. } \\
\text { - Retos y desafíos. }\end{array}$ & $\begin{array}{l}\text { Robótica con } \\
\text { Lego Mindstorm }\end{array}$ \\
\hline Albir-Mañanes (2014) & - Desarrollo de la creatividad. & - Retos y desafíos. & Robótica con Arduino \\
\hline Lizbardo (2015) & $\begin{array}{l}\text { - Aumento de la motivación. } \\
\text { - Mejora en los resultados académicos. }\end{array}$ & - Retos y desafíos. & Robótica con Arduino \\
\hline $\begin{array}{l}\text { Vega-Moreno Cufí, } \\
\text { Rueda y Llinás (2016) }\end{array}$ & $\begin{array}{l}\text { - Aumento de la motivación. } \\
\text { - Desarrollo de la creatividad. } \\
\text { - Favorecimiento de la transdisciplinariedad. } \\
\text { - Reducción del absentismo escolar }\end{array}$ & - Retos y desafíos. & Robótica con Arduino \\
\hline Tauler (2016) & $\begin{array}{l}\text { - Mejora de la gestión del aula } \\
\text { - Aumento de la motivación. } \\
\text { - Mejora de los resultados académicos. }\end{array}$ & $\begin{array}{l}\text { - Obtención de premios. } \\
\text { - Ranquin de puntos. }\end{array}$ & $\begin{array}{l}\text { Juego de rol educativo } \\
\text { ClassCraft }\end{array}$ \\
\hline Torres (2016) & $\begin{array}{l}\text { - Mejora de la gestión del aula. } \\
\text { - Aumento de la motivación. }\end{array}$ & $\begin{array}{l}\text { - Obtención de premios. } \\
\text { - Ranquin de puntos. }\end{array}$ & $\begin{array}{l}\text { Juego de rol educativo } \\
\text { ClassCraft }\end{array}$ \\
\hline $\begin{array}{l}\text { Gómez, Osorio y } \\
\text { Tapiero (2015) }\end{array}$ & - Mejora del trabajo colaborativo. & $\begin{array}{l}\text { - Obtención de premios. } \\
\text { - Ranquin de puntos. }\end{array}$ & $\begin{array}{l}\text { Juego de rol educativo } \\
\text { ClassCraft }\end{array}$ \\
\hline $\begin{array}{l}\text { Nieto, Heredia y } \\
\text { Cannon (2014) }\end{array}$ & - Desarrollo de la competencia matemática. & $\begin{array}{l}\text { - Obtención de premios. } \\
\text { - Ranquin de puntos. } \\
\text { - Feedback. }\end{array}$ & $\begin{array}{l}\text { Videojuego para consola } \\
\text { Xbox-Kinect }\end{array}$ \\
\hline Quintanal (2016) & $\begin{array}{l}\text { - Aumento de la motivación. } \\
\text { - Mejora de los resultados académicos. }\end{array}$ & $\begin{array}{l}\text {-Ranquin de puntos. } \\
\text {-Obtención de premios. }\end{array}$ & $\begin{array}{l}\text { Pizarra digital } \\
\text { Microsoft Office } \\
\text { Scratch }\end{array}$ \\
\hline $\begin{array}{l}\text { Saorin, De la Torre, } \\
\text { Melian, Meier y Rivero } \\
\text { (2015) }\end{array}$ & $\begin{array}{l}\text { - Aumento de la motivación. } \\
\text { - Desarrollo habilidades. Espaciales. } \\
\text { - Desarrollo de la competencia matemática. }\end{array}$ & $\begin{array}{l}\text { •Competición. } \\
\text { - Niveles. }\end{array}$ & $\begin{array}{l}\text { Videojuego para tabletas } \\
\text { Blockify } \\
\text { Impresión 3D }\end{array}$ \\
\hline
\end{tabular}

Fuente: elaboración propia. 
es protagonista y se ejemplifica el potencial de esta herramienta metodológica en el ámbito educativo de sTEM para la consecución de los objetivos docentes. En cada experiencia presentada se especifican cuáles son los elementos del juego empleados, los beneficios (documentados por los autores) que estos han aportado al proceso de enseñanza-aprendizaje y la tecnología (aplicación o dispositivo) con que se implementan dichos elementos.

Además de los beneficios que aporta la gamificación a la enseñanza de las materias STEM en secundaria, entre las que se encuentra un alumnado con frecuencia desmotivado (Marbà y Márquez, 2010), nos resulta de especial interés mostrar experiencias docentes que hacen uso de la gamificación y forman parte del acervo literario en el contexto de la educación superior, sobre todo, en áreas ligadas a la tecnología como, por ejemplo, la ingeniería-arquitectura (Lozada y Betancur, 2016). Esto corrobora que el binomio STEM-gamificación resulta exitoso en diferentes etapas educativas. De igual manera a como se ha hecho con las experiencias en secundaria, presentamos en la Tabla 4 las experiencias contextualizadas en el ámbito universitario.

\section{Resultados positivos de las experiencias educativas de gamificación en stem}

Las experiencias relacionadas en las tablas 3 y 4 reflejan cuáles son los beneficios de la introducción de la gamificación en el ámbito stem tanto en la secundaria con alumnado frecuentemente desmotivado como en la universidad, donde se requiere un nivel de profundización mayor de los contenidos académicos.

Elementos de los juegos tales como los puntos, los premios o las insignias que llevan a los jugadores (estudiantes en este caso) a superar niveles una vez alcanzan retos y desafíos en una competición se emplean, en las experiencias presentadas, a fin de conseguir objetivos docentes, como, por ejemplo, aumentar la motivación del alumnado, mejorar la gestión del aula, estimular el aprendizaje vivencial, favorecer tanto el desarrollo de la creatividad como de determinadas competencias y, en definitiva, mejorar los resultados académicos.

Al hacer un repaso general vemos cómo el aumento de la motivación se repite en la mayoría de las experiencias presentadas como un gran

Tabla 4. Resumen de las experiencias docentes analizadas en el ámbito universitario

\begin{tabular}{|c|c|c|c|}
\hline $\begin{array}{l}\text { Experiencias } \\
\text { documentadas }\end{array}$ & Beneficios en la enseñanza-aprendizaje & $\begin{array}{l}\text { Elementos de } \\
\text { gamificación }\end{array}$ & $\begin{array}{l}\text { Tecnología con que se } \\
\text { implementa }\end{array}$ \\
\hline Carpena et al. (2012) & $\begin{array}{l}\text {-Estimula el aprendizaje vivencial. } \\
\text {-Mejora de resultados académicos. }\end{array}$ & $\begin{array}{l}\text { - Competición. } \\
\text { - Ranquin de puntos. }\end{array}$ & Videojuegos para la consola wII. \\
\hline Cantador (2015) & $\begin{array}{l}\text {-Estimulación de la participación. } \\
\text {-Aumento de la motivación. } \\
\text { - Fomento del trabajo en equipo. } \\
\text {-Mejora de las relaciones interpersonales. } \\
\text {-Mejora de la comunicación oral efectiva. }\end{array}$ & $\begin{array}{l}\text { - Competición. } \\
\text { - Niveles. } \\
\text { - Retos y desafíos. } \\
\text { - Ranquin de puntos. }\end{array}$ & $\begin{array}{l}\text { Google Form } \\
\text { Matlab. }\end{array}$ \\
\hline $\begin{array}{l}\text { Astudillo, Bast y } \\
\text { Willging (2016) }\end{array}$ & $\begin{array}{l}\text {-Mejora de resultados académicos. } \\
\text { •Aumento de la motivación. }\end{array}$ & - Niveles. & $\begin{array}{l}\text { Serious games con Ligthbot, } \\
\text { Code.org y Scratch }\end{array}$ \\
\hline $\begin{array}{l}\text { Gértrudix y Gértrudix } \\
\text { (2013) }\end{array}$ & $\begin{array}{l}\text {-Ayuda a la transmisión de valores. } \\
\text {-Estimula el aprendizaje vivencial. } \\
\text {-Desarrollo de la creatividad. }\end{array}$ & $\begin{array}{l}\text {-Retos y desafíos. } \\
\text { •Competición. }\end{array}$ & $\begin{array}{l}\text { Videojuegos inmersivos } \\
\text { Minecraft y Sim Social }\end{array}$ \\
\hline $\begin{array}{l}\text { Uribe, Utrilla y } \\
\text { Santamaría (2017) }\end{array}$ & $\begin{array}{l}\text {-Ayuda a la asimilación de contenidos } \\
\text { (sustentabilidad). }\end{array}$ & •Competición. & $\begin{array}{l}\text { Serious games (juego } \\
\text { de mesa con trasfondo } \\
\text { académico). }\end{array}$ \\
\hline $\begin{array}{l}\text { Borrego, Fernández, } \\
\text { Robles y Blanes (2016) }\end{array}$ & $\begin{array}{l}\text { - Estimula el aprendizaje vivencial y significativo. } \\
\text {-Aumento de la motivación. }\end{array}$ & •Retos y desafíos. & $\begin{array}{l}\text { Escape room con } \\
\text { herramientas informáticas. }\end{array}$ \\
\hline Calderón et al. (2018) & $\begin{array}{l}\text {-Desarrollo de competencias personales y } \\
\text { habilidades sociales. }\end{array}$ & •Competición. & Serious games de simulación. \\
\hline
\end{tabular}

Fuente: elaboración propia. 
beneficio obtenido al introducir la gamificación en el ámbito educativo. Así, la búsqueda de la motivación juega un papel clave, por ejemplo, en la experiencia didáctica descrita por Murua (2013) y llevada a cabo en 15 centros de secundaria de Cataluña, el País Vasco y la Comunidad Valenciana. En esta, por medio de una red social online implementada con la aplicación Class Dojo, los alumnos de la asignatura de Matemáticas de cuarto grado de educación secundaria obligatoria (ESO) fueron evaluados usando un ranquin de puntos, obtenidos al realizar tareas de trigonometría divididas en niveles en los que consiguen poco a poco medallas o insignias. En la misma línea, Manzanares (2014) nos habla de cómo dotar al alumnado de secundaria de un mayor control sobre su aprendizaje, lo que repercute de forma positiva en la mejora, como decíamos, de la motivación, al gamificar la asignatura de Matemáticas de segundo grado de Eso con Classbadges, un sistema de acreditación de aprendizaje mediante insignias.

Además de la motivación, en secundaria importan otros aspectos tales como el desarrollo de competencias sociales e intelectuales del alumnado y la mejora tanto de la autoconfianza como de la satisfacción personal, a causa de su repercusión directa en el rendimiento académico. Así lo demuestran los resultados obtenidos en la experiencia descrita por Quintanal (2016) en la asignatura de Física y Química de cuarto grado de Eso de un centro educativo en Granada. En este caso, se planificó la gamificación de la asignatura para todo el curso realizando varios tipos de juegos competitivos, limitados en tiempo, en los que los alumnos debían contestar preguntas, completar listas de fórmulas y resolver problemas, tanto individualmente como por equipos, y obtener así premios que se traducen en puntos extra para el examen.

La experiencia vivencial y el aprendizaje significativo es lo que se persigue con el empleo de los videojuegos y la robótica en educación, lo cual gana cada vez más adeptos entre los docentes defensores del constructivismo (Vega y Cañas, 2014). Además, aunque la robótica puede también estar desligada de la gamificación, es habitual usar los tradicionales Lego o similares para fomentar la motivación y facilitar el aprendizaje de los estudiantes de electrónica, mecánica e informática, al dar solución a un problema tecnológico real (Ocaña et al., 2015). Esta nueva disciplina se incluye poco a poco en los planes educativos de centros tanto públicos como privados, ya que las áreas de conocimiento involucradas en los planteamientos de robótica forman parte del currículo de la secundaria y permiten aunar los contenidos de las asignaturas del ámbito STEM por medio de la creación práctica de robots programables con diferentes plataformas, como, por ejemplo, Scratch, Arduino o Lego Mindstorms, los cuales acometen determinadas tareas con un enfoque de ingeniería (Albir-Mañanes, 2014; Lizbardo, 2015; Vega y Cañas, 2014; Vega-Moreno et al., 2016) lo que permite promover la creatividad y el desarrollo del pensamiento computacional, habilidades que ayudarán al alumnado a enfrentarse a cualquier carrera universitaria del siglo xxi (Vázquez-Cano y Ferrer, 2015).

Además de estas experiencias que se centran, principalmente, en el aprendizaje de los contenidos de una materia, conviene comentar otras propuestas de gamificación que proponen gamificar la gestión del aula para contribuir a mejorar el ambiente de la clase y, por tanto, el rendimiento general del alumnado (García Velategui, 2015). A esto ayudan aplicaciones como Classcraft, un juego de rol educativo que se emplea en la experiencia que describe Tauler (2016) con resultados positivos en la mejora del comportamiento del alumnado, lo que incide directa y positivamente en el trabajo colaborativo (Gómez et al., 2015) y en la motivación (Torres, 2016).

Por otra parte, resulta de interés comentar que los aprendizajes del alumnado universitario del ámbito STEM, en los que se requiere un nivel de profundización mayor que en secundaria, también se ven favorecidos por la inclusión de la gamificación. Así se demuestra, por ejemplo, en la experiencia docente gamificada que describen Carpena et al. (2012), llevada a cabo en una universidad argentina, en la cual, a diferencia de años anteriores, se evidenció una mejora de los resultados académicos, ya que más del 85\% del alumnado aprobó de forma satisfactoria el curso de Artes multimediales I, al ser capaz de relacionar los conceptos extraídos de la bibliografía obligatoria de la asignatura con 
las experiencias lúdicas vividas en la clase usando la consola wir que, al incluir elementos del juego, como son los puntos y las clasificaciones, potencia la motivación de los estudiantes.

Conviene destacar que la superación de niveles, los badges, las insignias y las medallas implican un elemento de competición que genera controversia en el ámbito educativo (Melchor, 2012), pues no existe unanimidad sobre los beneficios o los perjuicios que se puedan derivar de él. Sin embargo, es indudable que se han documentado evidencias sobre su bondad (por ejemplo, en varias de las experiencias presentadas en las tablas 3 y 4). Así, Cantador (2015) analiza la influencia de la competición en una metodología gamificada para la asignatura de Introducción a la informática y la Programación en ordenador con sesenta estudiantes de Grado en Ingeniería Química de la Universidad Autónoma de Madrid. En su estudio demuestra que la competición no solo resulta beneficiosa para motivar a los estudiantes a esforzarse por alcanzar los objetivos marcados, sino que además estimula la participación (Moreno, Puentes, Ferrándiz, Flores y Acosta, 2014), fomenta el trabajo en equipo, las relaciones interpersonales y la adquisición de competencias transversales como, por ejemplo, la comunicación oral efectiva. Beneficios similares se documentan también en el desarrollo de competencias personales y habilidades sociales que contribuyen a la empleabilidad de futuros ingenieros, según Calderón et al. (2018), con dos serious games (“juegos serios") de simulación (Ball point game y Dealing with difficult people).

Por su parte, Martín del Pozo (2013) reflexiona sobre los jóvenes de hoy, los gamers (Astudillo, Bast y Willging, 2016), quienes dedican gran parte de su ocio a los videojuegos, lo cual se puede aprovechar en educación al crear mundos inmersivos abiertos en los que el alumnado se integra vivencialmente en el espacio y el tiempo del relato del juego (Gértrudix y Gértrudix, 2013). Estos videojuegos, además, facilitan la realización de simulaciones muy útiles en ciencias para conocer resultados de experimentos que serían costosos y complicados de realizar en las aulas (López, 2012), lo que permite al alumnado comprender cómo funciona el mundo y aprender a dar respuesta a problemas cotidianos (Luis-Pascual, 2015).

Los videojuegos pueden incluso fomentar la transmisión de valores y la responsabilidad social en el alumnado (Hernández y Cano, 2016) mediante experiencias que también podrían presentarse en el formato de escape room ("sala de escape") donde los participantes han de salir de una habitación tras sortear pruebas de ingenio, tal y como propone la experiencia de Borrego et al. (2016) con estudiantes del Grado en Ingeniería Informática de la Universidad Autónoma de Barcelona. El desafío, la exigencia, el interés y la diversión provocaron resultados positivos que animaron a que fuese ampliada en número de participantes y extendida a otras asignaturas.

Como se ha observado, la aplicación de metodologías gamificadas para el ámbito STEM aporta beneficios con los que los estudiantes se sienten más motivados e implicados en su propio aprendizaje, lo cual da como resultado una mejor asimilación de los conceptos abstractos, pues comprenden que estos están relacionados con fenómenos observables conectados con el mundo que los rodea, a la vez que les permiten desarrollar habilidades sociales y competencias transversales.

\section{Discusión y conclusiones}

A modo de recapitulación, destacamos que la sociedad actual se enfrenta hoy al reto de formar ciudadanos y ciudadanas con altos conocimientos del ámbito científico y tecnológico, con el objetivo de que sean protagonistas del futuro (Convert y Gugenheim, 2005). Sin embargo, los jóvenes de hoy en día sienten, por lo general, desapego por el estudio de materias de сут (Marbà y Márquez, 2010), en razón a la dificultad que encuentran para comprender los conceptos abstractos que forman la base del conocimiento científico-tecnológico, y porque no consiguen relacionarlos con su vida cotidiana. Por tanto, el movimiento stem persigue que la enseñanza de las materias de este ámbito, la cual, tradicionalmente, se realiza compartimentada (Von Garnier, 2010), se lleve a cabo de forma integrada en busca de un cambio de paradigma que acerque el conocimiento científico y tecnológico a 
los estudiantes y les cree motivación y curiosidad por entender el mundo que los rodea, así como los prepare para una sociedad tecnificada (Quingley et al., 2014).

Acercar el conocimiento de сут a los estudiantes de secundaria pasa por mejorar las metodologías de enseñanza en este ámbito en los centros educativos, por medio de experiencias vivenciales reales o simuladas que les permitan desarrollar el pensamiento crítico, resolver problemas de ingeniería y, por medio de la manipulación y construcción de objetos (López, 2012, Vega y Cañas, 2014), les ayuden a entender mejor conceptos que a priori resultan áridos, conectando con sus emociones y motivándolos a superarse. Es en esto que hemos visto cómo la gamificación puede resultar una opción provechosa, a tenor de las evidencias de la literatura. En efecto, la gamificación en el ámbito STEM estimula el aprendizaje del alumnado (Foncubierta y Rodríguez, 2006; Zichermann y Cunningham, 2011; Kapp, 2012; Díaz y Troyano, 2013) al introducir la mecánica de los juegos en contextos de no juego: crece la motivación de los aprendientes y, en consecuencia, también la implicación con el propio aprendizaje y los resultados académicos. Para esto se emplean elementos tales como los retos, la competición, la obtención de puntos, los ránquines, las insignias, los premios, etc. Asimismo, se obtienen beneficios, como, por ejemplo, el desarrollo de competencias (Calderón et al., 2018, Saorin et al., 2015), la mejora de la gestión del aula y el trabajo colaborativo (Borrego et al., 2016, Tauler, 2016; Torres, 2016; Gómez et al., 2015), el incremento de la motivación y la implicación del alumnado (Quintanal, 2016) o la mejor asimilación de contenidos (Uribe et al., 2017).

Aunque para gamificar se podría emplear el mínimo de tecnología, como se explica en la experiencia descrita por Quintanal (2016), es cierto que la mayoría de las propuestas didácticas analizadas en este artículo utilizan herramientas tecnológicas (Classcraft, ClassDojo, Xbox, Kinect, Blokify, consola Wii, Scratch, Arduino, Lego Mindstorm) a fin de conseguir una mayor implicación y motivación en los estudiantes que cursan la secundaria. Tal como afirma Sánchez-Aparicio
(2014), el alumnado siente fascinación por internet, los móviles y los videojuegos, y estas tecnologías de uso cotidiano permiten a los adolescentes comunicarse, relacionarse, divertirse y satisfacer su curiosidad (Machargo, Luján, León, López y Martín, 2003; Castellana, Sánchez-Carbonell, Graner y Beranuy, 2007).

Como se ha demostrado a lo largo del desarrollo argumentativo, aplicar una metodología gamificada con componente tecnológico en STEM proporciona la experiencia vivencial que permitirá a los estudiantes comprender y asimilar los contenidos de las materias del ámbito por medio de un aprendizaje significativo y de la participación activa (Da Silva, 2014), lo que resulta coherente con la sociedad tecnológica actual. En este sentido, Carpena et al. (2012) nos recuerdan que las instituciones educativas innovadoras muestran especial interés por una gamificación educativa que conecte los contenidos con experiencias prácticas. Así, además, al tener en cuenta el perfil de alumnado de secundaria, existe actualmente una importante tendencia que apuesta por el empleo en las aulas de videojuegos educativos para mejorar los procesos de enseñanza-aprendizaje (Marín, 2015), ya que estos proporcionan una dimensión vivencial que sumerge al jugador en una nueva vida virtual (Gértrudix y Gértrudix; 2013; Levis, 2003). No en vano, las metodologías gamificadas que emplean los videojuegos o la robótica (Albir-Mañanes, 2014; Lizbardo, 2015; Marín, 2015; Ocaña, 2012; Vega y Cañas, 2014; Vega-Moreno et al, 2016) persiguen los objetivos de los docentes STEM relacionados con el aprendizaje manipulativo y cooperativo, la aproximación a la resolución de problemas por medio de conjeturas o suposiciones, el uso integrado de la tecnología y el desarrollo del pensamiento crítico (Stohlmann et al., 2012).

En resumen, el potencial de la gamificación en el aula de stem, en especial al incluir el componente tecnológico, radica en aportar al alumnado una experiencia vivencial que le ayude a comprender mejor los contenidos curriculares de las distintas materias del ámbito y le haga conectar con sus propias emociones creándole interés, curiosidad y aprecio por el mundo en el que vive. Esta aportación positiva se refleja, como se ha evidenciado a lo largo de este artículo, en una mejoría significativa del 
desarrollo de sus capacidades, al fomentar el trabajo en equipo y ayudarle a sentirse motivado, al estimular su aprendizaje y promover el desarrollo de competencias transversales esenciales que proveen al alumnado de las herramientas necesarias para enfrentarse a los retos de la sociedad actual, desplegar así su creatividad y prepararlo para la vida.

En definitiva, como ya en el siglo xIx afirmó Pestalozzi (1986, p. 180), "el fin último de la educación no es la perfección en las tareas de la escuela, sino la preparación para la vida; no la adquisición de hábitos de obediencia ciega y de diligencia prescrita, sino una preparación para la acción independiente”. Por tanto, no hay mejor manera de conseguir esto que proporcionar a los estudiantes experiencias prácticas por medio de metodologías educativas innovadoras, como lo es la metodología gamificada con componente tecnológico para STEM que aquí se presenta, lo cual les ayudará a desarrollar sus capacidades y a entender mejor el mundo real, así como los prepara para adaptarse al medio social que se encontrarán en el futuro y llegar a ser ciudadanos activos comprometidos con la sociedad en la que viven.

\section{Apéndice A}

Tabla 1A. Artículos analizados más relevantes para esta investigación.

\begin{tabular}{|c|c|c|c|c|c|c|}
\hline & Documento & Editor & $\begin{array}{l}\text { Ámbito } \\
\text { educativo }\end{array}$ & $\begin{array}{l}\text { Área } \\
\text { conocimiento }\end{array}$ & $\begin{array}{l}\text { Tipo de } \\
\text { contribución }\end{array}$ & $\begin{array}{l}\text { Palabras clave } \\
\text { (si procede) }\end{array}$ \\
\hline 1 & Murua (2013) & $\begin{array}{l}\text { Universidad } \\
\text { Internacional de La } \\
\text { Rioja (España) }\end{array}$ & Secundaria & Matemáticas & $\begin{array}{l}\text { Trabajo de } \\
\text { investigación/tesis }\end{array}$ & $\begin{array}{l}\text { Recursos didácticos, } \\
\text { gamificación, motivación }\end{array}$ \\
\hline 2 & $\begin{array}{l}\text { Manzanares } \\
\text { (2014) }\end{array}$ & $\begin{array}{l}\text { Gobierno de Murcia } \\
\text { (España). Consejería } \\
\text { de Educación y Cultura }\end{array}$ & Secundaria & Matemáticas & $\begin{array}{l}\text { Descripción de } \\
\text { experiencia/ } \\
\text { informe }\end{array}$ & $\begin{array}{l}\text { Aprendizaje, matemáticas, } \\
\text { insignias, gamificación }\end{array}$ \\
\hline 3 & $\begin{array}{l}\text { García } \\
\text { Velategui } \\
(2015)\end{array}$ & $\begin{array}{l}\text { Universidad de } \\
\text { Cantabria (España) }\end{array}$ & Secundaria & $\begin{array}{l}\text { Gestión del } \\
\text { aula }\end{array}$ & $\begin{array}{l}\text { Trabajo de } \\
\text { investigación/tesis }\end{array}$ & $\begin{array}{l}\text { Gestión de Aula, } \\
\text { Gamificación }\end{array}$ \\
\hline 4 & $\begin{array}{l}\text { Vega y Cañas } \\
(2014)\end{array}$ & $\begin{array}{l}\text { Universidad Rey Juan } \\
\text { Carlos (España) }\end{array}$ & Secundaria & Tecnología & $\begin{array}{l}\text { Descripción de } \\
\text { experiencia/tesis }\end{array}$ & $\begin{array}{l}\text { Education, robotics, } \\
\text { teaching, constructivism }\end{array}$ \\
\hline 5 & $\begin{array}{l}\text { Ocaña, Romero, } \\
\text { Gily Codina } \\
(2015)\end{array}$ & $\begin{array}{l}\text { Investigación en la } \\
\text { escuela }\end{array}$ & Secundaria & Robótica & $\begin{array}{l}\text { Material curricular } \\
\text { y metodología } \\
\text { (descripción de } \\
\text { experiencia)/ } \\
\text { artículo }\end{array}$ & $\begin{array}{l}\text { Robótica educativa, } \\
\text { educación STEM, método } \\
\text { proyectos, materiales } \\
\text { curriculares }\end{array}$ \\
\hline 6 & $\begin{array}{l}\text { Albir-Mañanes } \\
(2014)\end{array}$ & $\begin{array}{l}\text { Universidad } \\
\text { Internacional de La } \\
\text { Rioja (España) }\end{array}$ & Secundaria & Robótica & $\begin{array}{l}\text { Trabajo de } \\
\text { investigación/tesis }\end{array}$ & $\begin{array}{l}\text { Robótica educativa, } \\
\text { creatividad, bachillerato, } \\
\text { química }\end{array}$ \\
\hline 7 & Lizbardo (2015) & $\begin{array}{l}\text { Encuentro Virtual } \\
\text { Educa }\end{array}$ & Secundaria & Robótica & $\begin{array}{l}\text { Descripción de } \\
\text { experiencia/ } \\
\text { comunicación }\end{array}$ & $\begin{array}{l}\text { Metodología de aprendizaje } \\
\text { inglove, robótica }\end{array}$ \\
\hline 8 & $\begin{array}{l}\text { Vega-Moreno, } \\
\text { Cufí, Rueda y } \\
\text { Llinás (2016) }\end{array}$ & $\begin{array}{l}\text { International Journal } \\
\text { of Educational } \\
\text { Research and } \\
\text { Innovation }\end{array}$ & Secundaria & Robótica & $\begin{array}{l}\text { Descripción de } \\
\text { experiencia/ } \\
\text { artículo }\end{array}$ & $\begin{array}{l}\text { Proyecto científico, } \\
\text { robótica, aprendizaje activo, } \\
\text { aprendizaje por experiencias }\end{array}$ \\
\hline 9 & Tauler (2016) & $\begin{array}{l}\text { Universitat Pompeu } \\
\text { Fabra (España) }\end{array}$ & Secundaria & $\begin{array}{l}\text { Gestión del } \\
\text { aula }\end{array}$ & $\begin{array}{l}\text { Trabajo de } \\
\text { investigación/tesis }\end{array}$ & $\begin{array}{l}\text { Gamificació, Classcraft, } \\
\text { motivació, aprenentatge, } \\
\text { Treball cooperatiu, atenció a } \\
\text { la diversitat. }\end{array}$ \\
\hline
\end{tabular}


Tabla 1A. Artículos analizados más relevantes para esta investigación.

\begin{tabular}{|c|c|c|c|c|c|c|}
\hline & Documento & Editor & $\begin{array}{l}\text { Ámbito } \\
\text { educativo }\end{array}$ & $\begin{array}{l}\text { Área } \\
\text { conocimiento }\end{array}$ & $\begin{array}{l}\text { Tipo de } \\
\text { contribución }\end{array}$ & $\begin{array}{l}\text { Palabras clave } \\
\text { (si procede) }\end{array}$ \\
\hline 10 & Torres (2016) & $\begin{array}{l}\text { Universitat de les Illes } \\
\text { Balears (España) }\end{array}$ & Secundaria & $\begin{array}{l}\text { Gestión del } \\
\text { aula }\end{array}$ & $\begin{array}{l}\text { Trabajo de } \\
\text { investigación/tesis }\end{array}$ & $\begin{array}{l}\text { Classcraft, motivació, } \\
\text { gamificació, tecnologia, } \\
\text { innovació educativa. }\end{array}$ \\
\hline 11 & $\begin{array}{l}\text { Gómez, Osorio } \\
\text { y Tapiero (2015) }\end{array}$ & $\begin{array}{l}\text { Fundación Los } \\
\text { Libertadores } \\
\text { (Colombia) }\end{array}$ & Secundaria & Informática & $\begin{array}{l}\text { Trabajo de } \\
\text { investigación/ } \\
\text { informe }\end{array}$ & $\begin{array}{l}\text { Trabajo colaborativo, } \\
\text { ludificación, ClassCraft, TIC } \\
\text { en educación }\end{array}$ \\
\hline 12 & $\begin{array}{l}\text { Nieto, Heredia } \\
\text { y Cannon } \\
(2014)\end{array}$ & Revista Relatec & Secundaria & Matemáticas & $\begin{array}{l}\text { Descripción de } \\
\text { experiencia/ } \\
\text { artículo }\end{array}$ & $\begin{array}{l}\text { Tecnología educativa, } \\
\text { videojuego, consola } \\
\text { de juego, habilidades } \\
\text { matemáticas }\end{array}$ \\
\hline 13 & $\begin{array}{l}\text { Quintanal } \\
\text { (2016) }\end{array}$ & eVsal Revistas & Secundaria & Física y química & $\begin{array}{l}\text { Descripción de } \\
\text { experiencia/ } \\
\text { artículo }\end{array}$ & $\begin{array}{l}\text { secundaria primer ciclo, } \\
\text { física, química,juego } \\
\text { educativo, motivación, } \\
\text { investigación educativa }\end{array}$ \\
\hline 14 & $\begin{array}{l}\text { Saorin, De la } \\
\text { Torre, Melian, } \\
\text { Meier y Rivero } \\
(2015)\end{array}$ & $\begin{array}{l}\text { Digital Education } \\
\text { Review }\end{array}$ & Secundaria & Tecnología & $\begin{array}{l}\text { Descripción de } \\
\text { experiencia/ } \\
\text { artículo }\end{array}$ & $\begin{array}{l}\text { Aplicaciones, tabletas } \\
\text { digitales, impresión 3D, } \\
\text { modelado 3D, educación, } \\
\text { juegos, vistas normalizadas }\end{array}$ \\
\hline 15 & $\begin{array}{l}\text { Carpena et al. } \\
\text { (2012) }\end{array}$ & $\begin{array}{l}\text { Sociedad } \\
\text { Iberoamericana de } \\
\text { Gráfica Digital,Sigradi }\end{array}$ & Universidad & $\begin{array}{l}\text { Artes } \\
\text { multimediales }\end{array}$ & $\begin{array}{l}\text { Descripción de } \\
\text { experiencia/ } \\
\text { artículo }\end{array}$ & $\begin{array}{l}\text { Docencia, metodologías, } \\
\text { juego, videojuegos, } \\
\text { gamificación }\end{array}$ \\
\hline 16 & $\begin{array}{l}\text { Cantador } \\
\text { (2015) }\end{array}$ & $\begin{array}{l}\text { Universidad } \\
\text { Autónoma de } \\
\text { Barcelona (España) }\end{array}$ & Universidad & Química & $\begin{array}{l}\text { Descripción de } \\
\text { experiencia/ } \\
\text { artículo }\end{array}$ & $\begin{array}{l}\text { Aprendizaje basado en } \\
\text { proyectos, competición, } \\
\text { gamificación }\end{array}$ \\
\hline 17 & $\begin{array}{l}\text { Astudillo, Bast } \\
\text { y Willging } \\
\text { (2016) }\end{array}$ & $\begin{array}{l}\text { Revista Virtualidad, } \\
\text { Educación y Ciencia }\end{array}$ & Universidad & Programación & $\begin{array}{l}\text { Trabajo de } \\
\text { Investigación/ } \\
\text { artículo }\end{array}$ & $\begin{array}{l}\text { Programación de } \\
\text { computadoras, gamificación, } \\
\text { juegos serios, estrategias } \\
\text { educativas }\end{array}$ \\
\hline 18 & $\begin{array}{l}\text { Gértrudix } \\
\text { y Gértrudix } \\
\text { (2013) }\end{array}$ & $\begin{array}{l}\text { Revista de Estudios de } \\
\text { Juventud }\end{array}$ & Universidad & TIC & $\begin{array}{l}\text { Trabajo de } \\
\text { Investigación/ } \\
\text { artículo }\end{array}$ & $\begin{array}{l}\text { Mundos inmersivos abiertos, } \\
\text { videojuegos de simulación } \\
\text { social, Sandbox, Minecraft, } \\
\text { Sim Social, educación, } \\
\text { aprendizaje }\end{array}$ \\
\hline 19 & $\begin{array}{l}\text { Uribe, Utrilla } \\
\text { y Santamaría } \\
\text { (2017) }\end{array}$ & $\begin{array}{l}\text { Revista Electrónica } \\
\text { sobre Educación } \\
\text { Media y Superior }\end{array}$ & Universidad & Arquitectura & $\begin{array}{l}\text { Descripción de } \\
\text { experiencia/ } \\
\text { artículo }\end{array}$ & $\begin{array}{l}\text { Sustentabilidad, diseño, } \\
\text { juegos de mesa, aprendizaje } \\
\text { basado en juegos, } \\
\text { aprendizaje significativo }\end{array}$ \\
\hline 20 & $\begin{array}{l}\text { Borrego, } \\
\text { Fernández, } \\
\text { Robles y Blanes } \\
(2016)\end{array}$ & Revista del Cidui & Universidad & Computación & $\begin{array}{l}\text { Descripción de } \\
\text { experiencia/ } \\
\text { artículo }\end{array}$ & $\begin{array}{l}\text { Room escape, enigmas, } \\
\text { gamificación, informática }\end{array}$ \\
\hline 21 & $\begin{array}{l}\text { Calderón et al. } \\
\text { (2018) }\end{array}$ & $\begin{array}{l}\text { Aenui, la Asociación } \\
\text { de Enseñantes } \\
\text { Universitarios de la } \\
\text { Informática (Palma de } \\
\text { Mallorca, España) }\end{array}$ & Universidad & Informática & $\begin{array}{l}\text { Descripción de } \\
\text { experiencia/ } \\
\text { artículo }\end{array}$ & $\begin{array}{l}\text { Competencias personales, } \\
\text { habilidades sociales, juegos } \\
\text { serios, evaluación de la } \\
\text { experiencia, ingeniería } \\
\text { informática }\end{array}$ \\
\hline
\end{tabular}

Fuente: elaboración propia. 


\section{Referencias}

Albir-Mañanes, M. (2014). La robótica aplicada a la asignatura de química de $1^{\circ}$ de bachillerato. Recuperado de: http://reunir.unir.net/handle/123456789/2716

Area, M. \& González, C. S. (2015). De la enseñanza con libros de texto al aprendizaje en espacios online gamificados. Educatio Siglo XXI, 33(3), 15-38. doi: $10.6018 / \mathrm{j} / 240791$

Astudillo, G. J., Bast, S. G. \& Willging, P. A. (2016). Enfoque basado en gamificación para el aprendizaje de un lenguaje de programación. Vitualidad, Educación y Ciencia, 12, 125-142. Recuperado de: https://revistas. unc.edu.ar/index.php/vesc/article/view/14739/14741

Bisquerra, R. (2009). Metodología de la investigación educativa. Madrid: Muralla.

Borrego, C., Fernández, C., Robles, S. \& Blanes, I. (2016). Room escape en las aulas: actividades de juegos de escape para facilitar la motivación y el aprendizaje de las ciencias de la computación. Revista del CIDUI, 3. Recuperado de: https://www.cidui.org/revistacidui/index. php/cidui/article/view/851

Calderón, A., Petri, G., Ruiz, M. \& Gresse, C. (2018). Desarrollando competencias personales y habilidades sociales en ingeniería informática mediante el uso de juegos serios. Actas de las Jornadas sobre Enseñanza Universitaria de la Informática, 3, 127-134. Recuperado de: http://www.aenui.net/ojs/index.php?journal $=$ actas_jenui\&page $=$ article $\& o p=$ viewArticle $\&$ path $\% 5 \mathrm{~B} \% 5 \mathrm{D}=467$

Cantador, I. (2015). La competición como mecánica de gamificación en el aula: una experiencia aplicando aprendizaje basado en problemas y aprendizaje cooperativo. En R. S. Contreras y J. L. Eguia (eds.), Gamificación en aulas universitarias (pp. 67-95). Barcelona: InCom-UAB. Recuperado de: http://incom.uab.cat/publicacions/edicion.asp? $\mathrm{id}=17$

Carpena, N., Cataldi \& M. Muñiz. G. (2012). En busca de nuevas metodologías y herramientas aplicables a la educación. Repensando nuestro rol docente en las aulas. Acta de la Conferencia Sigradi 2012, Fortaleza, Brasil, 633-635. Recuperado de: http://cumincades.scix.net/ data/works/att/sigradi2012_85.content.pdf

Castellana, M., Sánchez-Carbonell, X., Graner, C. \& Beranuy, M. (2007). El adolescente ante las tecnologías de la información y la comunicación: internet, móvil y videojuegos. Papeles Del Psicólogo, 38(3), 196-204. Recuperado de: https://www.redalyc.org/html/778/77828306/

Cheong, C., Filippou, J. \& Cheong, F. (2014). Towards the gamification of learning: investigating student perceptions of game elements. Journal of Information Systems
Education, 25(3), 233-244. Recuperado de: https:// pdfs.semanticscholar.org/2f57/df59f089fb37eb206b3c65754b3ad9eb8193.pdf

Consejo de Europa (2003). Conclusiones del Consejo de 5 de mayo de 2003 sobre los niveles de referencia del rendimiento medio europeo en educación y formación. Diario Oficial c134 de 7.6.2003. Recuperado de: http:// eur-lex.europa.eu/legal-content/ES/TXT/?uri=uriserv\%3Ac11064

Convert, B. \& Gugenheim, F. (2005). Scientific vocations in crisis in France: explanatory social developments and mechanism. European Journal Vocational Training, 35, 12-20.

Cortizo Pérez, J. C., Carrrero, F., Monsalve, B., Velasco. A., Días del Dedo, I. y Pérez, J. (2011). Gamificación y docencia: lo que la universidad tiene que aprender de los videojuegos. En VIII Jornadas Internacionales de Innovación Universitaria (pp. 1-8). Recuperado de: http://abacus.universidadeuropea.es/bitstream/handle/11268/1750/46_Gamificacion.pdf?sequence=2

Da Silva, E. (2014). Gamificación en la clase. En XI EVIDOSOL e VIII CILTE. Recuperado de: http://evidosol.textolivre. org/papers/2014/upload/16.pdf

Deterding, S. (2012). Gamification: designing for motivation. Interactions, 19(4), 14-17. doi: 10.1145/2212877.2212883

Díaz, J. \& Troyano, Y. (2013). El potencial de la gamificación en el ámbito educativo. III Jornadas de innovación docente de la Facultad de Ciencias de la Educación. "La innovación educativa: Respuesta en tiempos de incertidumbre”. Recuperado de https://idus.us.es/xmlui/ handle/11441/59067

Duque, M., Celis, J. \& Camacho, A. (2011). Cómo lograr alta calidad en la educación de los ingenieros: una visión sistémica. Revista Educación En Ingeniería, 6(12), 4860. Recuperado de: http://www.educacioneningenieria. org/index.php/edi/article/view/122

Ferguson, M. (1980). The acquarian conspiracy. Los Angeles: Tarcher.

Foncubierta, J. \& Rodríguez, C. (2006). Didáctica de la gamificación en la clase de español. Edinumen, 1-8. Recuperado de: http://www.edinumen.es/pdp14/Didactica_Gamificacion_ELE.pdf

García Velategui, A. (2015). Gestión de aula y gamificación. Utilización de elementos del juego para mejorar el clima en el aula. Recuperado de: https://repositorio.unican. es/xmlui/bitstream/handle/10902/7595/Garc\%C3\%ADaVelateguiAlejandro.pdf?sequence $=1$

Gértrudix, M. \& Gértrudix, F. (2013). Aprender jugando. Mundos inmersivos abiertos como espacios de aprendizaje de los y las jóvenes. Revista de Estudios de Juventud, 101, 123-137. Recuperado de: http://www.injuve. 
es/sites/default/files/2013/46/publicaciones/Documentos 9 Aprender jugando.pdf.

Gómez A. P., Osorio, D. A. y Tapiero, G. (2015). Fortalecimiento del trabajo colaborativo de los estudiantes del curso undécimo A, en el área de contabilidad-informática del colegio colombo japonés de Bogotá mediante la plataforma de ludificación Classcraft. Recuperado de: http://repository.libertadores.edu.co/handle/11371/256

González, P. \& López, N. (2011). La generación digital ante un nuevo modelo de televisión: contenidos y soportes preferidos. Anàlisi: Quaderns de Comunicació I Cultura, 44, 31-48. Recuperado de: http://www.raco.cat/ index.php/Analisi/article/view/248760

Hamari, J., Koivisto, J. \& Sarsa, H. (2014). Does gamification work? A literature review of empirical studies on gamification. Proceedings of the Annual Hawaii International Conference on System Sciences (HICSS) (pp. 3025-3034). Hawái: IEEE Computer Society. doi: 10.1109/HICSS.2014.377

Hernández, J. F. \& Cano, A. P. (2016). La transmisión de valores y responsabilidad social a partir de los videojuegos. Sphera Publica, 1(16), 114-131.

Herranz, E. \& Colomo-Palacios, R. (2012). La gamificación como agente de cambio en la ingeniería del software. Revista de Procesos y Métricas, 9(2), 30-56.

Kapp, K. (2012). The gamification of learning and instruction: game-based methods and strategies for training and education. San Francisco: John Wiley \& Sons.

Kischimoto, T. M. (1993). Jogos tradicionais Infantil: o jogo, a criança e a Educação. Petrópolis: Vozes.

Levis, D. (2003). Videojuegos en red: espacios simbólicos de juego y encuentro. En R. Aparici (coord.), Comunicación educativa en la sociedad de la información (pp. 1-14). Madrid: Universidad Nacional de Educación a Distancia. Recuperado de: http://diegolevis.com.ar/secciones/Articulos/videojuegos_Uned2003_VF.pdf

Lizbardo, B. (2015). Metodología de aprendizaje Inglove. En $x V$ Encuentro Virtual Educa. Recuperado de: http://reposital.cuaed.unam.mx:8080/jspui/handle/123456789/4074

López, F. (2012). Serious games. En Actas-IV Congreso Internacional Latina de Comunicación. La Laguna: Universidad de La Laguna. doi: 10.1109/VS-GAMES.2009.8

Lozada, C. \& Betancur, S. (2016). La gamificación en la educación superior: una revisión sistemática. Revista Ingenierías Universidad de Medellín, 16(31). doi: 10.22395/ rium.v16n31a5

Luis-Pascual, J. C. (2015). El juego auténtico y las claves de la gamificación del aprendizaje. En J. L. Bizelli, E. Sebastián Heredero y P. R. Marcal Riveiro (org.), Inclusao e aprendizagem: desafios para a escola em Ibero-América. San Pablo: Cultura Económica.

Machargo, J., Luján, I., León, M. E., López, P. \& Martín, M. A. (2003). Videojuegos por los adolescentes. Anuario de Filosofía, Psicología y Sociología, 6, 159-172.

Manzanares, S. (2014). Sistema de acreditación de aprendizajes basado en insignias (badges) en la materia de matemáticas. En J. Navarro, Mª D. Gracia, R. Lineros, y F. J. Soto (Coords.), Claves para una educación diversa. Murcia: Consejería de Educación y Cultura.

Marbà, A. \& Màrquez, C. (2010). ¿Qué opinan los estudiantes de las clases de ciencias? Un estudio transversal de sexto de primaria a cuarto de Eso. Enseñanza de Las Ciencias, 8, 19-30.

Marín, V. (2015). La gamificación educativa. Una alternativa para la enseñanza creativa. Digital Education Review, 27. Recuperado de: http://revistes.ub.edu/index. $\mathrm{php/der/article/view/13433/pdf}$

Martín del Pozo, M. (2013). Trabajar la educación para la salud en educación primaria con ayuda de videojuegos. En F. Ortega y L. Cardeñosa (eds.), III Congreso Internacional Comunicación 3.0. Las media enterprises y las industrias culturales, investigar la comunicación y los nuevos medios (pp. 291-304). Salamanca, España. Recuperado de: http://comunicacion3punto0.files. wordpress.com/2013/06/comunicacion3punto0libr oactas2012.pdf

Martín Martín, M. (2017). Aportaciones pedagógicas de las TIC a los estilos de aprendizaje. Tendencias Pedagógicas, 30. Recuperado de https://revistas.uam.es/tendenciaspedagogicas/article/view/8123

Melchor, E. (2012). Gamificacion y Elearning: un ejemplo con el juego pasapalabra. En 2012 EFQUEL Innovation Forum (pp. 1-6). Recuperado de: http://eif.efquel.org/ files/2012/09/ES_ES_EMelchorl.doc

Moreno, P. J., Puentes, C., Ferrándiz, E., Flores, E., \& Acosta, M. (2014). Trivianometrics, una competición académica por equipos en el aula de econometría financiera. Proyectos de Innovación y Mejora Docente, 1-4. Recuperado de: http://rodin.uca.es/xmlui/handle/10498/17406

Murua, E. (2013). Análisis de la gamificación como concepto aplicable en el proceso de enseñanza-aprendizaje de las matemáticas en 4o de Eso. Recuperado de: http:// reunir.unir.net/handle/123456789/2056

Nah F., Zeng Q., Telaprolu V., Ayyappa A. \& Eschenbrenner B. (22 de junio de 2014). Gamification of education: a review of literature. En HCI in Business. First International Conference (pp. 401-409). Heraklion: Springer.

Nieto, S. I., Heredia, Y. \& Cannon, B. Y. (2014). Xbox360 Kinect: herramienta tecnológica aplicada para el de- 
sarrollo de habilidades matemáticas básicas, en alumnos de segundo grado de Educación Básica en México. Relatec Revista Latinoamericana de Tecnología Educativa, 13(2), 103-117.

Ocaña, G. (2012). Robótica como asignatura en enseñanza secundaria. Resultados de una experiencia educativa. Espiral. Cuadernos del Profesorado, 5(10), 56-64. Recuperado de: http://www.cepcuevasolula.es/espiral/articulos/ESPIRAL_VOL_5_N_10_ART_5.pdf

Ocaña, G., Romero, I. M., Gil, F. \& Codina, A. (2015). Implantación de la nueva asignatura "Robótica" en Enseñanza Secundaria y Bachillerato. Investigación En La Escuela, 7(87), 65-79.

Ortiz-Colón, A.M., Jordán, J. \& Agredal, M. (2018). Gamificación en educación: una panorámica sobre el estado de la cuestión. Educação e Pesquisa, 44. doi: 10.1590/ s1678-4634201844173773

Ovallos, D., Villalobos, B., De la Hoz, S. \& Maldonado, D. (2016). Gamificación para la gestión de la innovación a nivel organizacional. Una revisión del estado del arte. Espacios, 37(8), 2-18.

Pérez-Manzano, A. \& Almela-Baeza, J. (2018). Gamificación transmedia para la divulgación científica y el fomento de vocaciones procientíficas en adolescentes. Comunicar, XXVI(55), 93-103.

Pestalozzi, J. H. (1986). Cómo Gertrudis enseña a sus hijos. Cartas sobre la educación de los niños. Libros de educación elemental. México: Porrúa.

Posada, F. (septiembre 9 de 2013). Gamificación educativa. Canaltic.com. Uso educativo de las TIC. Recuperado de: http://canaltic.com/blog/?p=1733\#aplica

Quigley, C. F., Herro, D. \& Jamil, F. M. (2014). Developing a conceptual model of STEAM teaching practices. School of Sciences and Mathematics, 117(1-2), 1-12.

Quintanal, F. (2016). Gamificación y la física-química de secundaria. evSAL Revistas, 17(3), 6-9. doi: 10.14201/ eks20161731328

Sánchez-Aparicio, J. C. (2014). Videojuegos y gamificación para motivar en educación. Andalucíaeduca, 120, 6-8. Recuperado de: http://www.andaluciaeduca.com/hemeroteca/ae_digital120.pdf

Saorin, J. L., De la Torre, J., Melian, D., Meier, C. \& Rivero, D. (2015). Blokify: juego de modelado e impresión 3D en tableta digital para el aprendizaje de vistas normalizadas y perspectiva. Digital Education Review, 27, 105-121. Recuperado de: dhttp://revistes.ub.edu/index. php/der/article/view/11651

Stohlmann, M., Moore, T. \& Roehring, G. (2012). Considerations for teaching integrated STEM education. Journal of Pre-College Engineering Education Research. doi: $10.5703 / 1288284314653$

Tauler, M. (2016). El classcraft com a mesura per incentivar la motivació, l'atenció a la diversitat i l'aprenentatge de les ciencies naturals. Recuperado de: https://repositori. upf.edu/handle/10230/27061

Torres, S. (2016). Classcraft: una proposta de treball a l'aula. Recuperado de: http://dspace.uib.es/xmlui/handle/11201/1815

Trujillo, F. (2012). Enseñanza basada en proyectos: una propuesta eficaz para el aprendizaje y el desarrollo de las competencias básicas. Revista Eufonía, 55(2010), 7-15.

Uribe, R. V., Utrilla, S. \& Santamaría, A. (2017). Aprendizaje basado en juegos. Una alternativa viable para la enseñanza significativa de la sustentabilidad. Revista Electrónica Sobre Educación Media Y Superior, 4(7).

Vázquez, A. \& Manassero, M. A. (2009). Patrones actitudinales de la vocación científica y tecnológica en chicas y chicos de secundaria. Revista Iberoamericana de Educación, 50(4), 1-15. Recuperado http://dialnet.unirioja. es/servlet/articulo?codigo=3055514\&info=resumen\&idioma $=\mathrm{SPA}$

Vázquez-Cano, E. \& Ferrer, D. (2015). La creación de videojuegos con Scratch en educación secundaria. Communication Papers. Media Literacy \& Gender Studies, 4(6), 63-73. Recuperado de: http://plataformarevistascomunicacion.org/2015/09/la-creacion-de-videojuegos-con-scratch-en-educacion-secundaria/

Vega, J. \& Cañas, J. M. (2014). Curso de robótica en educación secundaria usando constructivismo pedagógico. En Jornadas de Innovación y TIC Educativas-Jitice'14 Universidad Rey Juan Carlos. Móstoles, Madrid. Recuperado de: https://gsyc.urjc.es/jmplaza/papers/jitice2014.pdf

Vega-Moreno, D., Cufí, X., Rueda, M.J. \& Llinás, D. (2016). Integración de robótica educativa de bajo coste en el ámbito de la educación secundaria para fomentar el aprendizaje por proyectos. International Journal of Educational Research and Innovation (IJERI), 6, 162-175.

Von Garnier, C. (2010). La metamorfosis necesaria en la escuela. Sevilla: Ituci Siglo xxI.

Werbach, K. (2012). Gamification. Coursera.org. Recuperado de: https://www.coursera.org/course/gamification

Werbach, K. \& Hunter, D. (2012). For the win. Philadelphia: Wharton Digital Press.

Zichermann, G. \& Cunningham, C. (2011). Gamification by design: implementing game mechanics in web and mobile apps. Cambridge, MA: O’Reilly Media. 